\title{
Swiss Quality Award: les lauréats 2011
}

\author{
Cette année, plus de 100 projets ont été soumis en vue du Swiss Quality Award. Le \\ jury a récompensé les quatre meilleurs à l'occasion du quatrième Symposium natio- \\ nal pour la gestion de la qualité dans le secteur de la santé. La remise des prix, d'une \\ valeur de 10000 francs chacun, s'est déroulée le 12 mai 2011 à Berne.
}

\section{Jürg Beutlera, \\ Matthias Scholer ${ }^{b}$ \\ a Communication FMH \\ b Journaliste scientifique, vetcom.ch}

Correspondance:

FMH

Varja Nikolic

Données, démographie

et qualité (DDQ)

Elfenstrasse 18

Case postale 170

ddq@fmh.ch
CH-3000 Berne 15

La qualité ne tombe pas du ciel; elle est le fruit d'un travail acharné et d'un véritable engagement. C'est précisément cet engagement que le Swiss Quality Award entend encourager en récompensant des projets hors pair qui privilégient une approche innovante et portent un regard nouveau sur la gestion de la qualité. La quatrième édition du Swiss Quality Award témoigne de l'ampleur et de la diversité de cet engagement en Suisse: plus de 100 projets très prometteurs ont en effet été soumis dans les quatre catégories «Management», «Sécurité des pa-

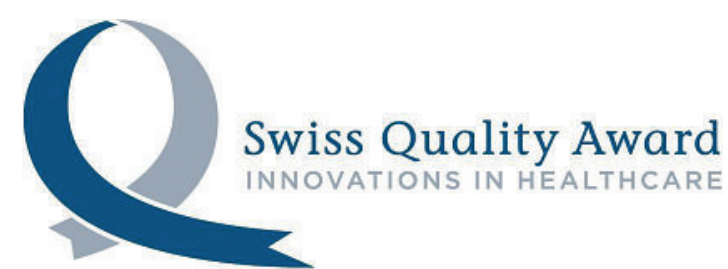

\section{Grande diversité}

Ce qui frappe d'emblée, c'est la grande diversité des innovations soumises. Elles couvrent les domaines les plus divers de la gestion de la qualité en médecine: innovations techniques, brochures destinées aux patients, pools de données pour des recommandations fondées sur des preuves, sites internet prodi-

guant des conseils aux patients, etc. Cette grande diversité se retrouve également dans les équipes à l'origine de ces innovations: celles-ci émanent tout aussi bien de cabinets tients», «Technologie» et «Information» - et plus de 30 ont été présentés, sous forme de posters, lors du quatrième Symposium national pour la gestion de la qualité dans le secteur de la santé.

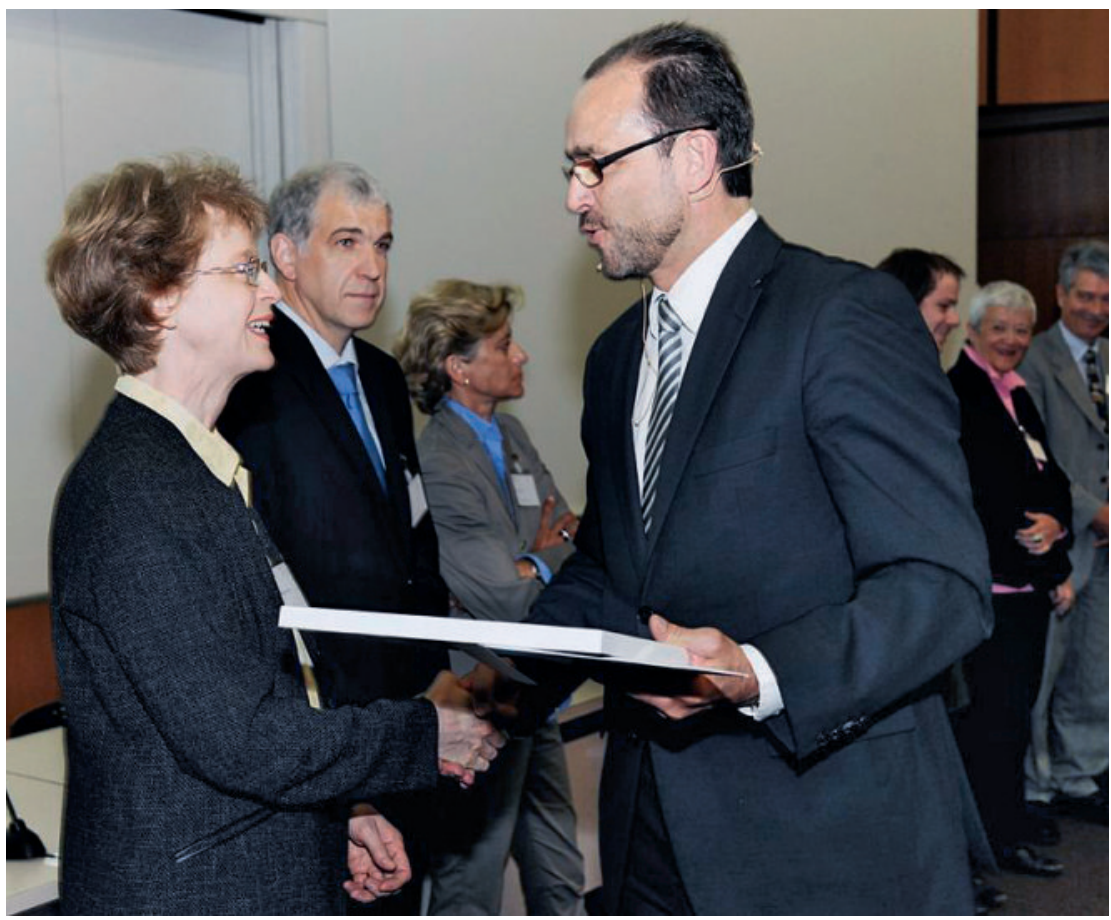

Remise des prix par Pascal Strupler, directeur de I'OFSP. médicaux, de cliniques universitaires, d'entreprises et d'organisations du domaine de la santé. Leur point commun: fournir des réponses à des problèmes concrets avec des projets qui ont déjà fait leurs preuves dans la pratique - un réel pas en avant pour les patients.

Pour évaluer ces innovations, les organisateurs du Swiss Quality Award ont désigné un jury spécialisé, composé également d'experts internationaux de la qualité. Tous les projets ont été anonymisés, puis évalués par plusieurs jurés sur la base d'une liste de critères transparents. Le jury a notamment tenu compte du degré d'innovation, de l'orientation clientèle ou encore de la durabilité.

\section{Les quatre projets primés}

Quatre innovations ont été particulièrement appréciées par le jury, qui a décidé à l'unanimité de leur décerner le Swiss Quality Award 2011. A l'origine de chacun de ces projets se trouve une équipe engagée, qui a mené son projet de l'idée de départ à la mise en œuvre finale. Le Prof. Philipp Schneider, président de la Société suisse pour le management de qualité dans la santé et coorganisateur du symposium, a salué ces «collaborateurs engagés, qui développent dans l'ombre des projets qui répondent à des besoins réels, et qui fournissent un travail exceptionnel». Le public a pu se rendre compte de l'énorme potentiel des innovations primées lorsque les chefs de projet ont présenté leur innovation au nom de toute leur équipe. 

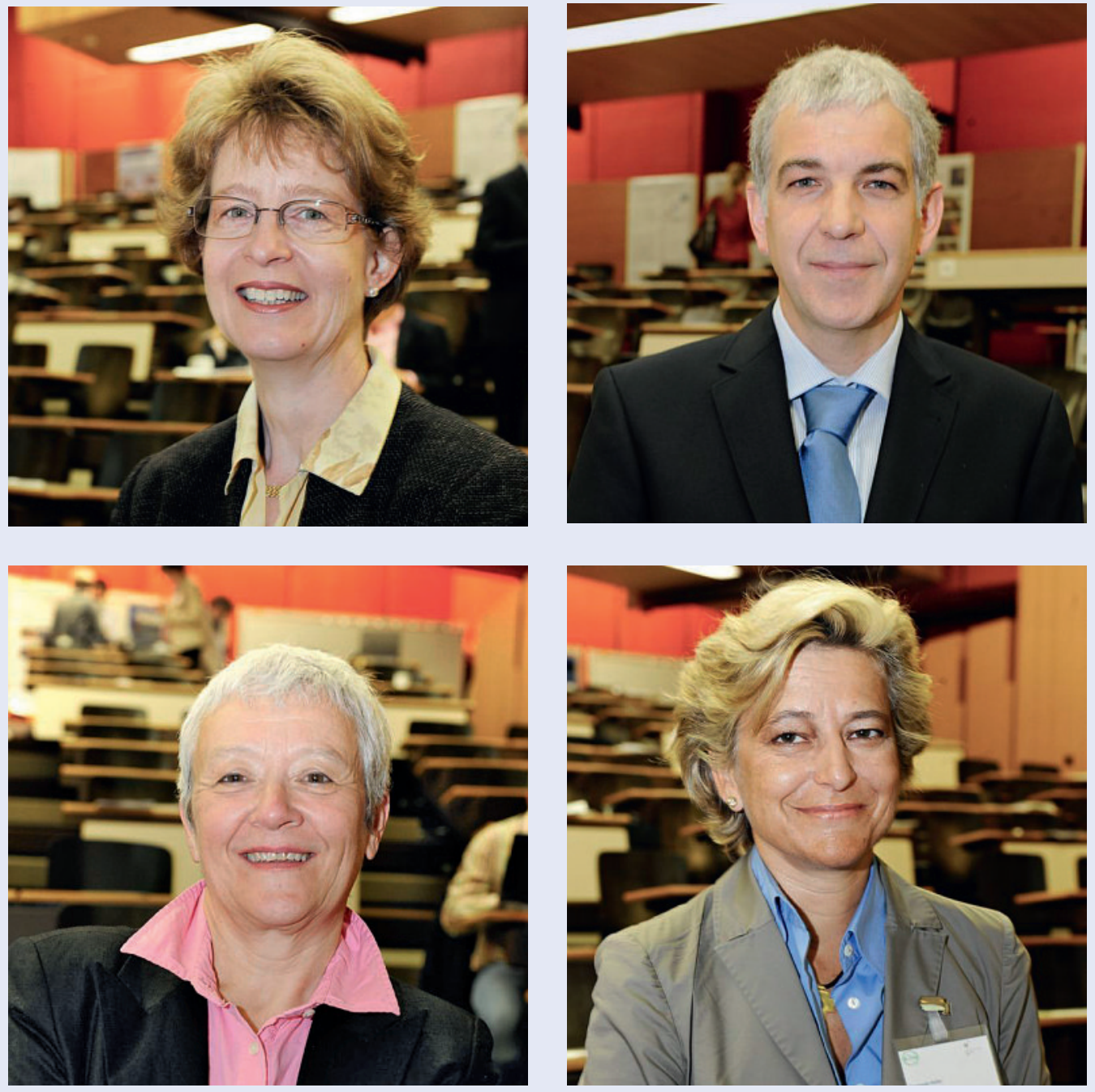

Les projets primés ont été présentés au nom des équipes impliquées par (dans le sens des aiguilles d'une montre): Eva Maria Genewein Depelteau, catégorie «Management»; Martin Egger, catégorie «Sécurité des patients»; Emanuela Keller, catégorie «Technologie»; Margareta Schmid, catégorie «lnformation».

\section{Catégorie «Management»}

Gestion du flux de patients aux urgences: maîtrise des pics de surcharge et réduction - de 60 à 10 par an - du nombre de goulots d'étranglement au service des urgences du centre hospitalier universitaire de Berne

Dans le passé, le service des urgences de l'hôpital de l'Ile (centre hospitalier universitaire) a dû fermer à plusieurs reprises pour cause de surcharge massive: une situation insatisfaisante pour toutes les personnes concernées. Grâce à des mesures appropriées, développées et introduites par un groupe de travail composé de médecins et de soignants, la fréquence des goulots d'étranglement a pu être diminuée de manière significative dans le service des urgences. L'expérience acquise à Berne peut également être mise à profit par d'autres centres d'urgence ou unités hospitalières.

\section{Catégorie «Sécurité des patients»}

Réduction des journées de sondage urinaire et des journées de traitement aux antibiotiques lors d'infection des voies urinaires en médecine hospitalière aiguë

Une étude scientifique de l'hôpital régional de l'Emmental et de l'Institut des maladies infectieuses de l'université de Berne montre qu'en renonçant sciemment à des interventions superflues dans certains domaines, on peut agir davantage pour la sécurité des patients qu'en recourant à des innovations technologiques onéreuses. 


\section{Catégorie «Technologie»}

Optical technologies for combined monitoring of cerebral blood flow and oxygen metabolism to improve outcome in patients with stroke, traumatic brain injury and after cardiac arrest Une équipe de chercheurs de l'Ecole polytechnique de Zurich et de l'hôpital universitaire de Zurich a développé deux méthodes pour mesurer de manière extrêmement précise l'oxygénation des régions cervicales endommagées. Ces innovations permettent de diminuer sensiblement le risque de séquelles après une attaque cérébrale ou un arrêt cardiaque.

\section{Catégorie «Information»}

Coaching santé - partenariat entre le patient et son médecin

Lorsque les patients décident de changer de mode de vie pour prendre soin de leur santé, ils peuvent compter sur un coaching taillé sur mesure de la part de leur médecin de famille. C'est, en résumé, l'objectif d'un nouveau programme mis au point par le Collège de médecine de premier recours pour renforcer la promotion de la santé et la prévention. Les premières expériences de la phase pilote réalisée dans le canton de Saint Gall sont extrêmement prometteuses.

\section{Grand honneur pour les lauréats}

Les quatre équipes lauréates ont non seulement reçu une somme de 10000 francs mais elles peuvent désormais utiliser l'appellation «Lauréat du Swiss Quality Award 2011». Lors de la cérémonie, Pascal
Strupler, directeur de l'Office fédéral de la santé publique (OFSP), s'est montré très impressionné par les projets: «Ces travaux nous confirment que la gestion de la qualité dans le domaine de la santé est loin d'être dans une impasse. Au contraire, ils représentent une motivation pour tous les acteurs de la santé de poursuivre leurs efforts.»

Le Swiss Quality Award est parrainé par la Fédération des médecins suisses (FMH), l'Institut pour la recherche évaluative en médecine (IEFM) de l'Université de Berne et la Société suisse pour le management de qualité dans la santé (SQMH). «Les patients dépendent des progrès en matière de gestion de la qualité. Le Swiss Quality Award récompense les projets qui s'engagent dans ce sens», déclare Daniel Herren, membre du Comité central de la FMH, au sujet de l'engagement de la FMH.

\section{Pour en savoir plus}

Le Swiss Quality Award est plus qu'une simple distinction: tous les projets présentés lors du Symposium national pour la gestion de la qualité dans le secteur de la santé du 12 mai 2011 sont également publiés sur www.swissqualityaward.ch ( $\rightarrow$ Les projets), permettant ainsi au public, spécialisé ou non, de s'informer. Un moteur de recherche permet de retrouver le projet souhaité.

Un résumé des quatre projets primés paraîtra dans les deux prochains numéros du Bulletin des médecins suisses. 